\title{
Microvascular decompression of the cochleovestibular nerve for treatment of tinnitus and vertigo: a systematic review and meta-analysis of individual patient data
}

\author{
Minke J. C. van den Berge, MD, ${ }^{1,3}$ J. Marc C. van Dijk, MD, PhD, ${ }^{2}$ Iris A. Posthumus, MD, ${ }^{1}$ \\ Nynke Smidt, MD, PhD, ${ }^{4,5}$ Pim van Dijk, PhD, ${ }^{1,3}$ and Rolien H. Free, MD, PhD ${ }^{1,3}$
}

Departments of ${ }^{1}$ Otorhinolaryngology/Head and Neck Surgery, ${ }^{2}$ Neurosurgery, ${ }^{4}$ Epidemiology, and ${ }^{5}$ Geriatrics, University Medical Center Groningen; and ${ }^{3}$ Graduate School of Medical Sciences (Research School of Behavioral and Cognitive Neurosciences), University of Groningen, The Netherlands

\begin{abstract}
OBJECTIVE Microvascular decompression (MVD) is regarded as a valid treatment modality in neurovascular conflicts (NVCs) causing, for example, trigeminal neuralgia and hemifacial spasms. An NVC of the cochleovestibular nerve might cause tinnitus and/or vertigo; however, general acceptance of MVD for this indication is lacking. The aim of this study was to investigate the effectiveness, safety, and prognostic factors for success of MVD of the cochleovestibular nerve.

METHODS A systematic review and meta-analysis of individual patient data (IPD) were conducted according to the Preferred Reporting Items for Systematic Reviews and Meta-Analyses and Individual Patient Data (PRISMA-IPD) guidelines. By a comprehensive search (conducted in January 2016) in MEDLINE, EMBASE, and Google Scholar, eligible studies were identified. The collected outcome was a global measurement of improvement of 1) tinnitus, 2) vertigo, and 3) tinnitus combined with vertigo. For the meta-analysis, IPD were collected from the papers and/or from the authors. IPD were analyzed with logistic regression analysis while accounting for study clustering.
\end{abstract}

RESULTS Thirty-five studies (572 patients) were included. The level of evidence provided by these studies was low. In $28 \%$ of patients with tinnitus and $32 \%$ of patients with vertigo, complete relief following MVD was reported. Patients with both tinnitus and vertigo had complete relief in $62 \%$ of cases. In $11 \%$ of patients, $\geq 1$ complications were reported. Metaanalysis of IPD (165 patients) demonstrated that patients with both tinnitus and vertigo had a higher chance of success (OR 3.8, 95\% $\mathrm{Cl} 1.45-10.10)$ than patients with tinnitus alone. No other variables were significantly related to success.

CONCLUSIONS Due to low success rates, MVD cannot be considered as a standard treatment method for tinnitus or vertigo. Moreover, a substantial complication rate was found. However, patients with combined symptoms had a higher chance of success. When combined symptoms occur, it is more likely that an NVC is the underlying pathology and MVD might be appropriate. Due to the low level of evidence in the included studies, this conclusion must be taken with caution. Further validation is necessary to evaluate whether patients with combined symptoms are indeed better candidates for MVD.

https://thejns.org/doi/abs/10.3171/2016.8.JNS16992

KEY WORDS cochleovestibular nerve; microvascular decompression; neurovascular conflict; tinnitus; vertigo; functional neurosurgery

$\mathrm{A}$ NEUROVASCULAR conflict (NVC) is a well-known neurological phenomenon, in which the root entry zone of a cranial nerve is compressed by an artery or vein. As such, an NVC may cause symptoms related to the affected nerve. In 1932, neurosurgeon Walter Dandy was the first to propose this concept, describing an NVC of the trigeminal nerve in the posterior fossa as the cause of trigeminal neuralgia. ${ }^{10}$ In the late 1960 s, the theory of NVC received more attention after the publication by Peter Jannetta of a large series of microvascular decompression (MVD) surgeries as treatment for symptomatic NVC of various cranial nerves. ${ }^{19}$

Today, MVD surgery is a widely accepted treatment for a symptomatic NVC of the trigeminal nerve (i.e., trigemi-

ABBREVIATIONS ABR = auditory brainstem response; IPD = individual patient data; IQR = interquartile range; $M V D=$ microvascular decompression; $\mathrm{NVC}=$ neurovascular conflict; PRISMA = Preferred Reporting Items for Systematic Reviews and Meta-Analyses.

SUBMITTED April 18, 2016. ACCEPTED August 18, 2016.

INCLUDE WHEN CITING Published online December 2, 2016; DOI: 10.3171/2016.8.JNS16992. 
nal neuralgia), facial nerve (i.e., hemifacial spasms), and glossopharyngeal nerve (i.e., glossopharyngeal neuralgia) ${ }^{21,31,48}$ It has been suggested that NVC of the cochleovestibular nerve could be a cause of unilateral tinnitus and vertigo. ${ }^{19}$ An NVC of the cochleovestibular nerve may cause a heterogeneous symptomatology, because the nerve is composed of the superior vestibular nerve, the inferior vestibular nerve, and the cochlear nerve. Therefore, compression of the cochleovestibular nerve has the potential to cause symptoms of tinnitus and/or vertigo, sometimes accompanied by sensorineural hearing loss, which in the literature is also referred to as the cochleovestibular nerve compression syndrome. ${ }^{41}$

Unlike MVD for trigeminal neuralgia, for example, general acceptance of MVD for tinnitus and/or vertigo is lacking. For trigeminal neuralgia, the success rate at long-term follow-up is $83 \% .^{48}$ For hemifacial spasms and glossopharyngeal neuralgia, the success rates $(91 \%$ and $92 \%-98 \%$, respectively) are even higher. ${ }^{21,31}$ In contrast, the estimated success rate of MVD for tinnitus is between $28 \%$ and $100 \%$, and for vertigo it is between $75 \%$ and $100 \% .{ }^{47}$ This dissimilarity in success rates may be caused by the lack of sufficient diagnostic criteria for tinnitus and/or vertigo caused by an NVC, resulting in inadequate patient selection.

To tackle the ongoing controversy regarding this type of surgery, more insight is needed. The results of many studies in which MVD was performed for tinnitus and/or vertigo have been published since 1975 . However, to our knowledge, no meta-analysis of these data has been performed. Therefore, we conducted a systematic review and meta-analysis of individual patient data (IPD) in all studies, assessing the effectiveness of MVD of the cochleovestibular nerve for patients with complaints of tinnitus and/ or vertigo. In addition, complication rates and prognostic factors of success were reviewed, to gain more insight into safety and adequate patient selection.

\section{Methods}

This systematic review and the IPD meta-analysis were conducted according to the methods of the Cochrane Collaboration ${ }^{17}$ and the Preferred Reporting Items for Systematic Reviews and Meta-Analyses and Individual Patient Data (PRISMA-IPD) guidelines. ${ }^{42} \mathrm{~A}$ protocol for this systematic review was specified in advance and published in the PROSPERO database (CRD42015017437) (www.crd. york.ac.uk/PROSPERO).

\section{Eligibility Criteria and Information Sources}

A systematic search in MEDLINE (PubMed) and EMBASE was conducted on February 18, 2015, and was updated on January 27, 2016. The search strategy was developed using the PICO method. The "P" (participants) were patients with an NVC of the cochleovestibular nerve and symptoms of tinnitus and/or vertigo. The "I" (intervention) was MVD surgery of the cochleovestibular nerve. The " $\mathrm{C}$ " (comparison) and "O" (outcome) were left open to assure a broad search. The peer-reviewed search strategy was designed using the following search indexing terms: "tinnitus," "vertigo," "cochleovestibular nerve compression syndrome," "microvascular decompression," "cochleoves- tibular nerve," and other variations. An additional search was conducted in Google Scholar, to avoid missing articles that lacked one of the search terms in the title, abstract, or index terms. In addition, the Cochrane Central Register of Controlled Trials was searched for relevant articles. Furthermore, references of all included studies and relevant reviews on this topic were screened for potentially eligible studies.

\section{Study Selection}

Eligible articles consisted of studies that met the following criteria: 1) included patients with an NVC of the cochleovestibular nerve with symptoms of tinnitus and/or vertigo; 2) investigated the effectiveness of MVD of the cochleovestibular nerve; 3 ) included a measure of recovery as outcome; and 4) presented the results in a quantitative way. Only full-text, peer-reviewed papers were included in the systematic review. No restrictions were made with regard to the design of the study. Publications written in languages other than English, Dutch, or German were excluded. The study selection was performed by 2 reviewers (M.J.C.B. and I.A.P.) independently. Disagreements between reviewers were resolved in a consensus meeting. All retrieved titles were screened for eligibility, followed by screening of the remaining abstracts. Subsequently, a final selection was made based on the full-text papers. When it was suspected that papers were based on the same study population (i.e., same study or same study center), the paper with the most complete patient data or, if papers were equally complete, the paper with the longest follow-up data was included. Other overlapping studies were excluded.

\section{Data Extraction and Methodological Quality Assessment}

A predesigned form was used to extract data from the included studies. Data extraction was performed by 1 reviewer (M.J.C.B.) and was cross-checked by another (I.A.P.). The following information was extracted both on a study level and on an individual patient level (if available): 1) patient characteristics (age, sex, symptoms, duration of symptoms before surgery, specification of symptoms, auditory brainstem response [ABR], preoperative use of carbamazepine for symptom relief); 2) inclusion criteria, type of intervention, causative vessel identified perioperatively; 3 ) length of follow-up, recurrence of symptoms, necessity of reoperation; 4) primary outcome (i.e., global measurement of improvement of preoperative symptoms) indicating the treatment success; and 5) complications. For each study, information on complications was registered and categorized as minor complications (e.g., transient facial palsy, CSF leakage, wound infection, transient hearing deficit) or major complications (permanent facial palsy, permanent hearing deficit, meningitis, stroke, death). If no IPD were reported in the article, the corresponding author of the included study was contacted by email with a request to provide the (additional) IPD. After 2 and 4 weeks, a reminder was sent.

The methodological quality of all included studies was assessed by 2 reviewers (M.J.C.B. and I.A.P.) independently, using the "Quality Assessment Tool for Case Series Studies" (adjusted for the research topic) from the National 
Institutes of Health (https://www.nhlbi.nih.gov/health-pro/ guidelines/in-develop/cardiovascular-risk-reduction/tools/ case_series). Follow-up was regarded as adequate if the mean follow-up was $\geq 1$ year. Disagreements between the reviewers were discussed and resolved in a consensus meeting. If necessary, the final decision was made by a third reviewer (N.S.). The overall percentage of agreement and Cohen's kappa were calculated to evaluate interrater agreement on the methodological quality of the included studies. To provide insight into possible publication bias, a scatterplot of sample size of study against percentage of complete relief of symptoms was constructed.

\section{Statistical Analysis \\ Aggregate Data Analysis}

For all included studies, the mean data on a study level (aggregate data) were presented using descriptive statistics. To quantitatively analyze the data, the postoperative outcome (i.e., global measurement of improvement) was categorized into 4 groups: "complete relief" (i.e., symptom free); "improvement" (defined as any variation of improvement); "no change"; and "worsening." The outcome was related to change in preoperative symptoms of 1) tinnitus and 2) vertigo. Because there was also a proportion of patients in whom symptoms of both occurred, a subgroup analysis was conducted for patients with 1) tinnitus, 2 ) vertigo, and 3 ) vertigo and tinnitus. The overall treatment outcome was presented as a percentage, calculated by the number of patients with, for example, complete relief of tinnitus divided by the total number of patients who underwent MVD for, in this case, tinnitus.

\section{Individual Patient Data Meta-Analysis}

To investigate prognostic variables of success of MVD, a meta-analysis of IPD was performed. For this purpose, the primary outcome (i.e., success of MVD surgery) was dichotomized into 2 categories: 1) success (defined as complete relief of symptoms and marked improvement of symptoms); and 2) no success (defined as minimal improvement of symptoms, no change, or worsening). The IPD from all studies were analyzed using a binary logistic regression model (1-stage approach), while accounting for clustering among patients within the same study by including random study effects. ${ }^{1,42}$ Odds ratios, $95 \%$ CIs, and overall $\mathrm{p}$ values are presented. An OR $>1$ indicates increased odds for success of the intervention. Continuous variables (e.g., age, follow-up) were described using means with standard deviation for normal distributed data and median and interquartile range (IQR) for skewed distributed data. All statistical analyses were performed using IBM SPSS Statistics (version 22). Differences with a p value < 0.05 were regarded as significant.

\section{Results \\ Study Selection}

The initial search retrieved 1167 articles (MEDLINE: 550, EMBASE: 610, Google Scholar: 7, Cochrane Central Register of Controlled Trials: 0). Duplicate articles were removed. Review of 803 titles, 255 abstracts, and 92 fulltext articles resulted in the inclusion of 35 articles..$^{2-6,8}$, 13-16,18-20,22,23,25,27-30,33,34,35-41,43-47,49 Special care was taken to avoid including studies with potentially overlapping participants (16 studies were excluded for this reason). For an overview of the selection process, see the PRISMA-IPD flow diagram (Fig. 1). An update of the search, using the same search strategy and selection process, was performed on January 27, 2016, and identified 34 additional articles. None of these articles were eligible for inclusion.

\section{Characteristics of 35 Studies Comprising Total Population of Review}

Details regarding characteristics of the included studies are presented in Table 1 . The 35 studies included in this review were published between 1980 and 2015 and originated from various countries. The included studies were case reports or case series, and the number of enrolled patients per study varied from 1 to 163 . In total, 572 patients were included in this review, of whom 313 (55\%) were women. As the main symptom, 207 (36\%) patients had tinnitus, $222(39 \%)$ had vertigo, and $143(25 \%)$ had both tinnitus and vertigo. The mean age at surgery was $52 \pm 6.7$ years. The median duration of symptoms prior to surgery was 48 months (IQR 26-74 months) and the median follow-up was 19 months (IQR 9-38 months).

Abnormal ABR measurements preoperatively were reported in 312 of 398 (78\%) patients. Not all studies specified the criteria for abnormality of ABR; however, most studies reported a prolonged wave I-III interval. Indications for performing MVD varied among the studies, as shown in Table 1. Recurrence of preoperative complaints was described in 35 of 446 (8\%) patients, followed by revision MVD in 31 (7\%) patients (data not shown). The vessel most often reported as the cause of the NVC was the anterior inferior cerebellar artery (in 16\% of cases); however, in $60 \%$ of cases the causative vessel was not reported (data not shown).

\section{Assessment of Study Quality}

The interrater agreement on the methodological quality assessment was substantial (overall agreement $81 \%$ [227 of 280]; Cohen's kappa 0.63). ${ }^{24}$ Results of the methodological quality assessment of the included studies are presented in Fig. 2. Two studies were conducted prospectively ${ }^{22,36}$ and 33 retrospectively. The majority of the studies were case series $(\mathrm{n}=22)$ and the other studies were case reports $(\mathrm{n}=$ 13), i.e., a description of only 1 patient. The most frequently encountered flaw was that the "outcome measure was not clearly defined, valid, reliable, and/or implemented consistently" (Fig. 2). Only 4 (11\%) studies scored positive on this item..$^{6,15,22,45}$

\section{Treatment Success of MVD: Aggregate Data Analysis}

The outcomes of MVD on a study level are described in an outcome table (see Appendix). The aggregate data analysis shows that complete relief of symptoms following MVD was achieved in $28 \%$ of patients with tinnitus and in $32 \%$ of patients with vertigo (Fig. 3, upper). In a second 


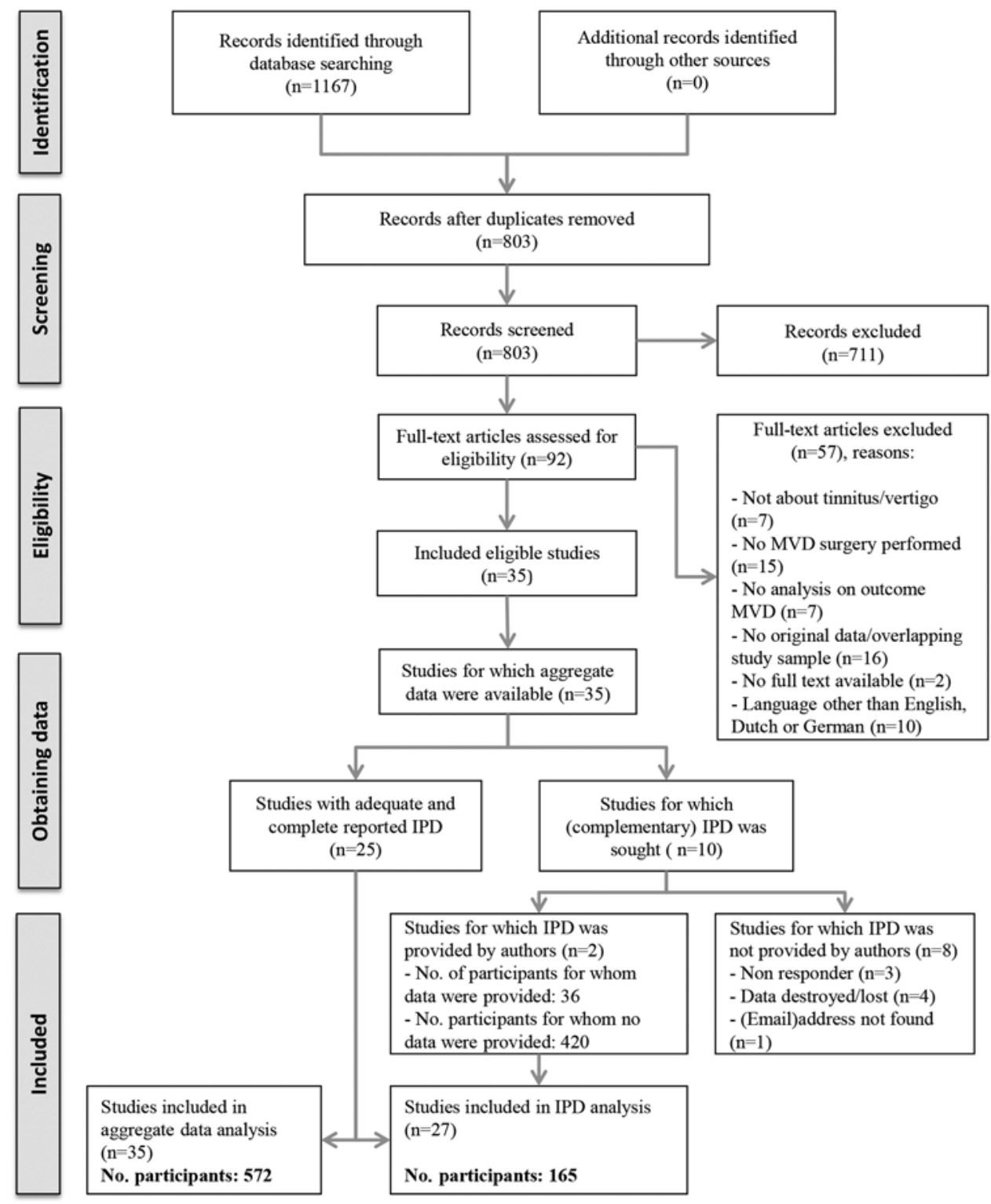

FIG. 1. Flow diagram of study selection process according to PRISMA-IPD.

analysis, the outcomes were split among "tinnitus," "vertigo," and "tinnitus and vertigo." In this analysis, there was treatment success in $62 \%$ of patients who had both tinnitus and vertigo, in $22 \%$ of patients with tinnitus, and in $27 \%$ of patients with vertigo (Fig. 3, lower).

\section{Meta-Analysis of Individual Patient Data}

Individual patient data were adequately reported in 25 studies. ${ }^{2,4,5,8,13-16,18,19,23,25,27-30,35,37-40,43-47}$ From the remaining 10 studies, IPD were requested. This resulted in the inclusion of IPD from 2 more studies in the IPD analysis ${ }^{3,36}$ (Fig. 1). Finally, IPD from 165 patients were available, originating from 27 studies (marked with an asterisk in Table 1). In Table 2, patients' characteristics are shown for the "success" group $(\mathrm{n}=108)$ and the "no success" group $(n=57)$. For each of the 27 studies, Table 3 shows which terminology was used to define "success" versus "no success." For several variables, few data were available (e.g., "Type of tinnitus symptoms" [n = 21], "Type of vertigo symptoms" [ $\mathrm{n}=24]$, and "Successful use of carbamazepine" $[n=69])$. For these variables, a univariate analysis was not appropriate; therefore, they were excluded from the analysis.

Results of the univariate binary logistic regression analysis of the remaining variables (Table 4) demonstrated that patients with both tinnitus and vertigo had a higher chance of "success" compared with patients with only tinnitus ( $\mathrm{p}$ $<0.001$, OR 3.8, 95\% CI 1.45-10.10). Patients who underwent the translabyrinthine route of surgery had a lower chance of treatment success $(\mathrm{p}=0.01$, OR $0.14,95 \%$ CI 0.04-0.50) compared with the most frequently used retrosigmoidal approach. No other variables were significantly 


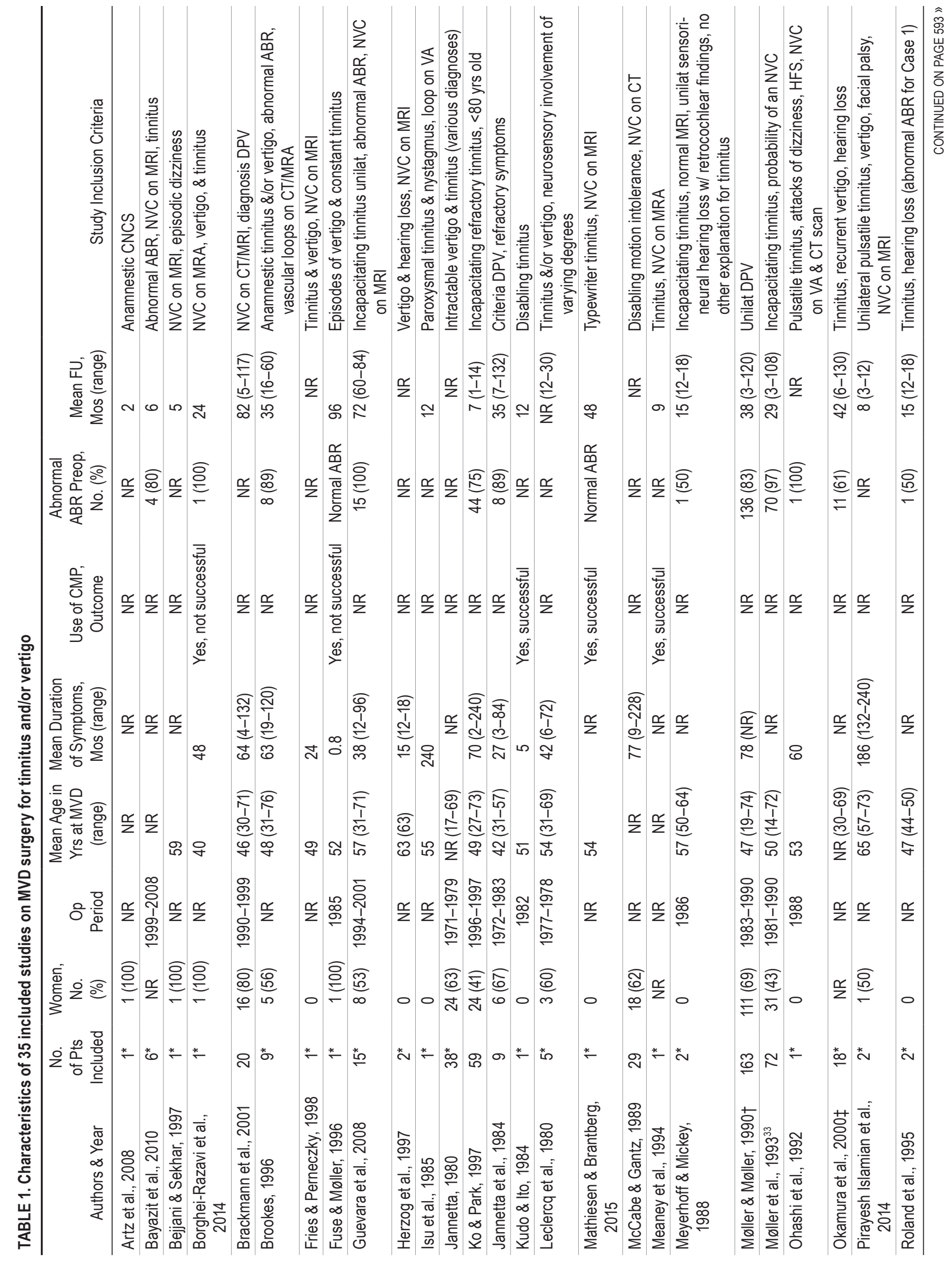




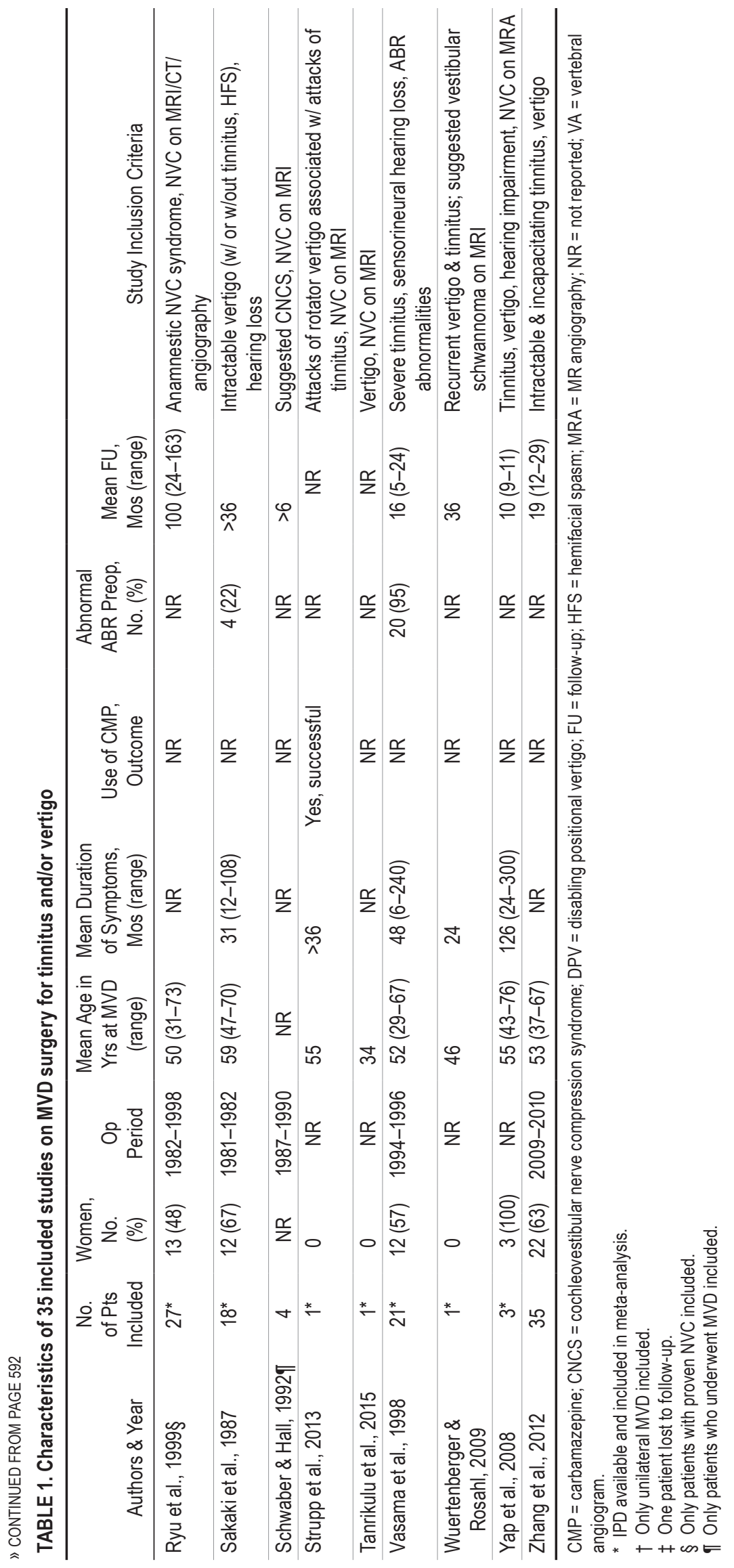


Q1. Was the study question or objective clearly stated?

Q2. Was the study population clearly and fully described, including case definition?

Q3. Were the cases consecutive?

Q4. Was the intervention and causative vessel clearly described?

Q5. Were the outcome measures clearly defined, valid, reliable and implemented consistently?

Q6. Was the length of follow-up adequate? (>1 yr average)

Q7. Were the statistical methods well-described?

Q8. Were the results well-described?
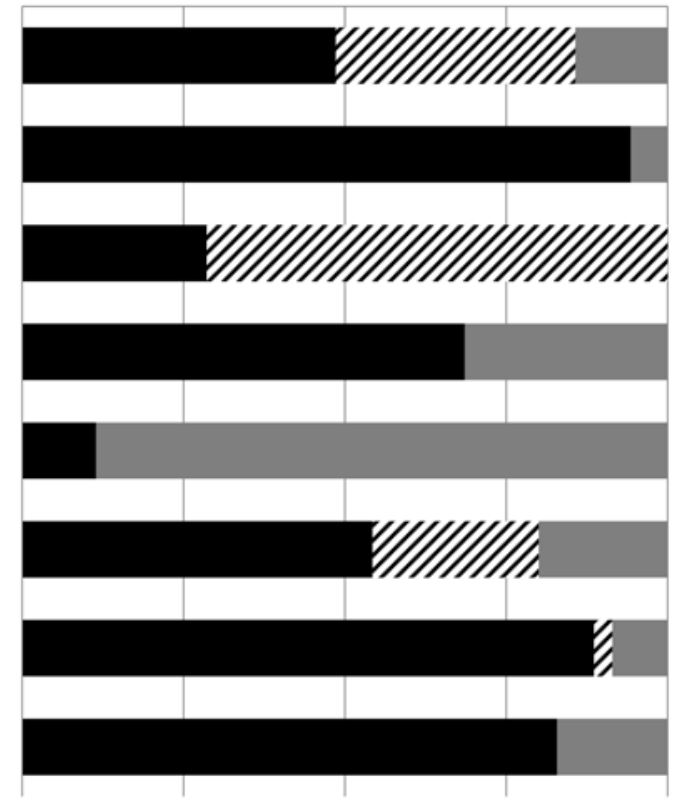

$0 \%$
$25 \%$
$75 \%$

$100 \%$

Yes \# Not Applicable/Not reported No

FIG. 2. Assessment of study quality for included studies using the National Institutes of Health: Quality Assessment Tool for Case Series Studies (adjusted for this topic).

related to treatment "success" or "no success." No multivariate logistic regression analysis could be performed because there were too many missing values for the total number of variables and sample size.

\section{Surgical Complications}

Thirteen of the 35 included articles did not report complications. An analysis of complications from the remaining 22 studies (representing 492 patients) is presented in Fig. 4. Minor complications were reported in $3 \%$ of patients and major complications in $6 \%$. The most common complication was permanent hearing deficit after surgery (5\% of patients). Overall, $11 \%$ of patients had complications following MVD. No strokes or deaths were registered.

\section{Assessment of Publication Bias}

Figure 5 shows a scatterplot of "sample size of study" versus "complete relief (of all symptoms)." In the smaller studies (i.e., $\mathrm{n}<40$ ), high as well as low success rates were published. This suggests that there is no severe risk of publication bias. However, no formal statistical tests for publication bias could be performed on these data.

\section{Discussion}

\section{Summary of Evidence}

This systematic review and meta-analysis assessed the treatment success of MVD of the cochleovestibular nerve for tinnitus and/or vertigo. The success rate, defined as the percentage of patients who had complete relief, was $28 \%$ for patients with tinnitus and $32 \%$ for patients with vertigo. If patients had both tinnitus and vertigo, treatment success was $62 \%$. The meta-analysis of IPD also showed that patients with tinnitus combined with vertigo had a higher chance of treatment success than patients with only tinnitus or only vertigo. Moreover, a substantial complication rate was encountered. No other prognostic factors related to age, sex, preoperative $\mathrm{ABR}$, duration of symptoms, symptom specification, or use of carbamazepine could be identified.

In 2008, Yap et al. attempted to identify the success rate of MVD of the cochleovestibular nerve in a systematic review and found a very wide range of success (defined as "complete relief and/or marked improvement") of 28\%$100 \%$ for tinnitus and $75 \%-100 \%$ for vertigo. ${ }^{47}$ Yap et al. included 22 studies without the guidance of PRISMA, whereas our systematic review comprises 35 studies. Our review provides a more specific analysis of treatment success, subdivided into 4 outcome categories. By evaluating the percentage of "complete relief" instead of "complete relief and/or marked improvement," we were able to make a comparison with success rates of MVD for other cranial nerves, such as in trigeminal neuralgia. In this review, a complication rate of $11 \%$ was encountered, which is more specific than that of Yap et al., who reported that "morbidity was minimal."'47

The rate of "complete relief" from MVD for tinnitus or vertigo is low compared with the success rates of MVD for other cranial nerves. ${ }^{21,31,49}$ One of the reasons for this rather low success rate might be the fact that an NVC of the cochleovestibular nerve can cause a wide variety of symptoms, sometimes resembling other diagnoses such as 

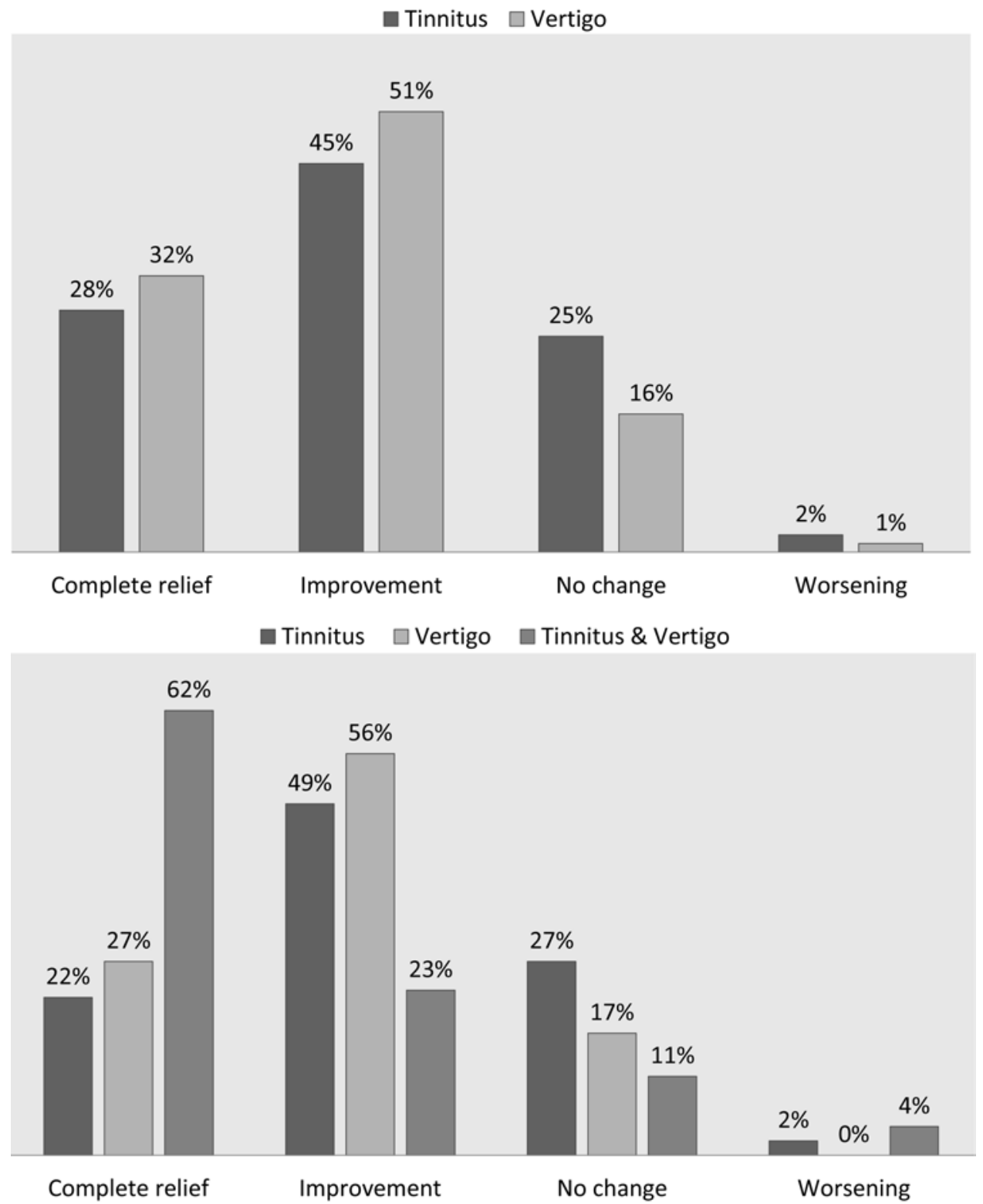

FIG. 3. Overview of surgical outcomes for tinnitus and vertigo after MVD surgery of the cochleovestibular nerve, for "tinnitus" and "vertigo" (upper) and for "tinnitus," "vertigo," and "tinnitus \& vertigo" (lower).

Ménière's disease. Therefore, it is challenging to correctly assign tinnitus and/or vertigo complaints to an NVC. Several studies have attempted to specify a typical patient group with tinnitus and/or vertigo that would benefit from MVD. ${ }^{11,27,33,34,41}$ However, presenting symptoms of NVC of the cochleovestibular nerve are not as distinct as those of trigeminal neuralgia, for example, which makes it difficult to determine adequate selection criteria.

Nevertheless, this review showed that if patients had both tinnitus and vertigo, the success rate increased to $62 \%$. This remarkable difference, compared with the success rate of tinnitus alone or vertigo alone, suggests that when both symptoms occur in 1 patient, the underlying pathology is more likely to be of an NVC and thus MVD is an appropriate treatment. The cochleovestibular nerve contains both a vestibular and a cochlear branch; conflict of a vessel might therefore affect both nerve branches and may cause symptoms related to both tinnitus and vertigo. This hypothesis is supported by findings of Ryu et al., who correlated the complaints of an NVC to the exact location of compression on the nerve. ${ }^{39}$

It must be noted that other inner ear disorders may cause tinnitus combined with vertigo, such as Ménière's disease. Therefore, other likely causes must be excluded, and additional information is needed to make the presumption that an NVC is the cause of the complaints. For example, an MRI with an NVC on the ipsilateral side of the complaints would point to an NVC, although it has been reported that some patients have an NVC on MRI but do not have any complaints. ${ }^{9}$ However, the likelihood of an NVC as cause 
TABLE 2. Characteristics of patients included in the IPD meta-analysis and differences between the "Success" and "No Success" groups

\begin{tabular}{|c|c|c|c|}
\hline Characteristic & Total Population, N = 165 & Success, $n=108$ & No Success, $n=57$ \\
\hline \multicolumn{4}{|l|}{ Sex } \\
\hline M & $71 / 149(48)$ & 49/104 (47) & $22 / 45(49)$ \\
\hline $\mathrm{F}$ & $78 / 149(52)$ & $55 / 104(53)$ & $23 / 45(51)$ \\
\hline Mean age $\pm S D$, yrs $(n=148)$ & $52 \pm 12$ & $51 \pm 12$ & $53 \pm 10$ \\
\hline \multicolumn{4}{|l|}{ Preop symptoms } \\
\hline Tinnitus & $75 / 165(46)$ & $35 / 108(32)$ & $40 / 57(70)$ \\
\hline Vertigo & $16 / 165(10)$ & 16/108 (15) & 0 \\
\hline Tinnitus \& vertigo & $74 / 165(45)$ & $57 / 108(53)$ & $17 / 57(30)$ \\
\hline \multicolumn{4}{|l|}{ Type of tinnitus symptoms } \\
\hline Pulsatile & $6 / 21(28)$ & 6/18 (33) & 0 \\
\hline Nonpulsatile & $9 / 21(43)$ & $6 / 18(33)$ & $3 / 3(100)$ \\
\hline Paroxysms & 6/21 (28) & 6/18 (33) & 0 \\
\hline \multicolumn{4}{|l|}{ Type of vertigo symptoms } \\
\hline Paroxysms & $8 / 24(33)$ & $6 / 22(27)$ & $2 / 2(100)$ \\
\hline Constant & $1 / 24(4)$ & $1 / 22(5)$ & 0 \\
\hline DPV & $13 / 24(54)$ & $13 / 22(59)$ & 0 \\
\hline Other & $2 / 24(8)$ & $2 / 22(9)$ & 0 \\
\hline \multicolumn{4}{|l|}{ Duration of symptoms, yrs } \\
\hline $0-2$ & $36 / 114(32)$ & $26 / 76(34)$ & 10/38 (26) \\
\hline$>2-4$ & $29 / 114(25)$ & $20 / 76(26)$ & $9 / 38(24)$ \\
\hline$>4$ & 49/114 (43) & $30 / 76(40)$ & $19 / 38(50)$ \\
\hline \multicolumn{4}{|l|}{ Successful use of carbamazepine } \\
\hline Successful use & $3 / 69(4)$ & $3 / 40(8)$ & 0 \\
\hline No successful use & $33 / 69(48)$ & $26 / 40(65)$ & $7 / 29(24)$ \\
\hline Success NR & $2 / 69(3)$ & $1 / 40(2)$ & $1 / 29(3)$ \\
\hline No use & $31 / 69(45)$ & $10 / 40(25)$ & $21 / 29(73)$ \\
\hline \multicolumn{4}{|l|}{ Route of op } \\
\hline Retrosigmoidal & 48/111 (43) & $36 / 77(47)$ & $12 / 34(35)$ \\
\hline Retrolabyrinthine & $4 / 111(4)$ & $3 / 77(4)$ & $1 / 34(3)$ \\
\hline Retromastoidal & $37 / 111(33)$ & $28 / 77(36)$ & 9/34 (26) \\
\hline Translabyrinthine & $17 / 111(15)$ & $5 / 77(6)$ & $12 / 34(35)$ \\
\hline Suboccipital & $5 / 111(5)$ & $5 / 77(6)$ & 0 \\
\hline \multicolumn{4}{|l|}{ Causative vessel } \\
\hline AICA & 78/151 (52) & $51 / 100(51)$ & $27 / 51(53)$ \\
\hline PICA & $11 / 151(7)$ & $7 / 100(7)$ & $4 / 51(8)$ \\
\hline Vertebral artery & $11 / 151(7)$ & $8 / 100(8)$ & $3 / 51(6)$ \\
\hline Combination & 39/151 (26) & $25 / 100(25)$ & $14 / 51(27)$ \\
\hline Other & 12/151 (8) & $9 / 100(9)$ & $3 / 51(6)$ \\
\hline \multicolumn{4}{|l|}{ Preop ABR } \\
\hline Abnormal & $72 / 95(76)$ & $42 / 53(79)$ & $30 / 42(71)$ \\
\hline Normal & 23/95 (24) & $11 / 53(21)$ & $12 / 42(29)$ \\
\hline Median FU in yrs, $n=155$ (IQR) & $2.0(1-4.8)$ & $1.8(1-3.5)$ & $2.5(1.0-5.0)$ \\
\hline
\end{tabular}

$\mathrm{AICA}=$ anterior inferior cerebellar artery; PICA = posterior inferior cerebellar artery.

All values are reported as the number of patients $(\%)$, unless otherwise indicated.

of the complaints might increase when several clues add up. Clinical findings such as changes in ABR; anamnestic unilateral, paroxysmal attacks of tinnitus or vertigo; and responsiveness to carbamazepine have been suggested as other selection criteria. ${ }^{11,26,27,32}$ Unfortunately, in our IPD analysis, none of these suggested selection criteria could be confirmed.

The patient group included in the IPD analysis was rep- 
TABLE 3. Definition of "Success" and "No Success" for IPD meta-analysis

\begin{tabular}{|c|c|c|}
\hline Authors \& Year & "Success" & "No Success" \\
\hline Artz et al., 2008 & "Resolved" & - \\
\hline Bayazit et al., 2010 & "Complete relief" \& "partial relief" & "Worsening of tinnitus" \\
\hline Bejjani \& Sekhar, 1997 & "Complete relief" & - \\
\hline Borghei-Razavi et al., 2014 & "Complete relief" & - \\
\hline Brookes, 1996 & $\begin{array}{l}\text { "Complete abolishment," "cured," \& "reduction in } \\
\text { objectified tinnitus loudness" }\end{array}$ & "No benefit" \& "no significant reduction in tinnitus loudness" \\
\hline Fries \& Perneczky, 1998 & $\begin{array}{l}\text { "Persistent relief from vertigo" \& "partial relief from } \\
\text { tinnitus" }\end{array}$ & - \\
\hline Fuse \& Møller, 1996 & - & "First improved, then increasing symptoms of vertigo \& tinnitus" \\
\hline Guevara et al., 2008 & "Totally free" \& "improved" & "No change" \& "worse" \\
\hline Herzog et al., 1997 & "Symptoms resolved," "symptoms subsided" & - \\
\hline Isu et al., 1985 & "Completely relieved" & - \\
\hline Jannetta, 1980 & "No symptoms," "no vertigo, slight tinnitus" & - \\
\hline Kudo \& Ito, 1984 & "Much reduced, returned to work" & - \\
\hline Leclercq et al., 1980 & "Good result" \& "returned to work" & "Returned to preop level" \\
\hline Mathiesen \& Brantberg, 2015 & "Relief from attacks" & - \\
\hline Meaney et al., 1994 & "Complete resolution" & - \\
\hline Meyerhoff \& Mickey, 1988 & "Almost totally subsided" \& "marked improvement" & - \\
\hline Ohashi et al., 1992 & "Symptoms disappeared" & - \\
\hline Okamura et al., $2000^{*}$ & $\begin{array}{l}\text { "Free of vertigo," "recovered tinnitus w/ low-pitched } \\
\text { tinnitus," "marked recovered of vertigo" }\end{array}$ & $\begin{array}{l}\text { "Remained low-pitched tinnitus," "improvement of vertigo \& } \\
\text { remained tinnitus" }\end{array}$ \\
\hline Pirayesh Islamian et al., 2014 & $\begin{array}{l}\text { "Symptoms alleviated" \& "completely free of } \\
\text { symptoms" }\end{array}$ & - \\
\hline Roland et al., 1995 & "Almost complete relief" \& "improved markedly" & - \\
\hline Ryu et al., $1999 \dagger$ & "Improved" and "resolved" & "Symptoms present" \\
\hline Sakaki et al., 1987 & $\begin{array}{l}\text { "Free," "markedly improved," \& "moderately } \\
\text { improved" }\end{array}$ & "Mildly improved" \& "unchanged" \\
\hline Strupp et al., 2013 & "No symptoms" & - \\
\hline Tanrikulu et al., 2015 & "Symptoms diminished" & - \\
\hline Vasama et al., 1998 & "Totally free" \& "markedly improved" & "Slightly improved," "unchanged," \& "worse" \\
\hline Wuertenberger \& Rosahl, 2009 & "Resolved completely" & - \\
\hline Yap et al., 2008 & "Completely abolished" & "Slight improvement" \\
\hline
\end{tabular}

If outcomes of tinnitus and vertigo were presented separately, the following apply: if tinnitus was unchanged and vertigo resolved (or otherwise) = no success; if tinnitus improved and vertigo resolved (or otherwise) = success; if both improved = success; if tinnitus was unchanged and vertigo improved (or otherwise) = no success.

* One patient lost to follow-up.

† Only patients with proven NVC included.

resentative of the total MVD population from the aggregate data analysis, in terms of sex, age, duration of symptoms, and follow-up. In accordance with the outcomes of our aggregate data analysis, the IPD analysis showed that if MVD was performed in patients with both tinnitus and vertigo, there was a significantly higher chance of treatment success compared with patients with tinnitus alone. Also, it was shown that the translabyrinthine approach for MVD resulted in statistically significantly lower treatment success, which suggests that this surgical approach should not be used. Indeed, this approach seems obsolete to use in patients with tinnitus and vertigo, because in this method the vestibule and semicircular canals are surgically removed and complete sensorineural hearing loss is induced, which in turn may cause tinnitus and vertigo.

It was found that $78 \%$ of the reported preoperative $\mathrm{ABR}$ measurements were abnormal. De Ridder et al. proposed a pathophysiological mechanism for tinnitus resulting from NVC of the cochleovestibular nerve. They reported that if a blood vessel causes an NVC with the auditory part of the nerve, then a disorganized signal transmission occurs (objectified by Peak II decrease in ABR testing), resulting in tinnitus. ${ }^{11}$ However, although ABR abnormalities have been suggested to result from an NVC of the cochleovestibular nerve, our IPD analysis did not demonstrate that abnormality of $\mathrm{ABR}$ is a prognostic factor for treatment success.

The preoperative duration of symptoms has also been suggested to be related to the outcome of MVD..$^{12,33}$ De Ridder et al. argued that in an NVC of the cochleovestibular nerve, tinnitus is initially the result of impaired signal transmission at the level of the vascular contact. ${ }^{12}$ The lon- 
TABLE 4. Univariate logistic regression analysis with prognostic factors for "Success"

\begin{tabular}{|c|c|c|c|}
\hline Variable & OR & $95 \% \mathrm{Cl}$ & $\mathrm{p}$ Value \\
\hline Sex & & & 0.77 \\
\hline M & Ref & & \\
\hline $\mathrm{F}$ & 1.07 & $0.65-1.76$ & \\
\hline Age & 0.99 & $0.96-1.01$ & 0.25 \\
\hline Preop symptoms & & & 0.000 \\
\hline Tinnitus & Ref & & \\
\hline Vertigo & NA & NA & \\
\hline Tinnitus \& vertigo & 3.83 & $1.45-10.10$ & \\
\hline Duration of symptoms, yrs & & & 0.30 \\
\hline $0-2$ & Ref & & \\
\hline$>2-4$ & 0.86 & $0.38-1.92$ & \\
\hline$>4$ & 0.61 & $0.29-1.28$ & \\
\hline Route of op & & & 0.01 \\
\hline Retrosigmoidal & Ref & & \\
\hline Retrolabyrinthine & 1.00 & $1.00-1.00$ & \\
\hline Retromastoidal & 1.04 & $0.37-2.90$ & \\
\hline Translabyrinthine & 0.14 & $0.04-0.50$ & \\
\hline Suboccipital & NA & NA & \\
\hline Causative vessel & & & 0.89 \\
\hline AICA & Ref & & \\
\hline PICA & 0.93 & $0.31-2.75$ & \\
\hline Vertebral artery & 1.42 & $0.36-5.55$ & \\
\hline Combination & 0.95 & $0.36-2.50$ & \\
\hline Other & 1.59 & $0.39-6.43$ & \\
\hline Preop ABR & & & 0.43 \\
\hline Normal & Ref & & \\
\hline Abnormal & 1.53 & $0.46-5.05$ & \\
\hline FU & 0.97 & $0.86-1.09$ & 0.59 \\
\hline
\end{tabular}

$\mathrm{NA}=$ not available; Ref $=$ reference.

Overall $p$ values are presented.

ger the compression exists, the more damage is done to the auditory nerve, which may lead to demyelination of the nerve. In turn, this may translate to differentiation of auditory input into the central auditory cortex, leading to tinnitus. Therefore, De Ridder et al. suggested that surgical decompression should be performed within 4 years after the onset of symptoms. ${ }^{12}$

In our IPD analysis, no statistically significant relationship between the preoperative symptom duration and a successful outcome could be demonstrated. Finally, several authors have suggested that the specification of symptoms is essential in diagnosing a symptomatic NVC, e.g., so-called typewriter tinnitus. Typewriter tinnitus is a clicking or ticking noise that may occur in paroxysms of tinnitus and may be combined with ipsilateral vestibular symptoms, and it has been suggested to result from an NVC. ${ }^{7,26}$ Unfortunately, the specification of preoperative symptoms was underreported in these IPD; therefore, this information could not be included in a statistical model. In future research, more attention must be paid to this topic, because specification of symptoms might be essential in identifying those patients who may benefit from MVD.

\section{Limitations of the Study}

This systematic review has limitations that merit emphasis. First, an important finding is that the only studies available that addressed our research topic had low levels of evidence (Level 4). This must be kept in mind when interpreting the presented results. On the other hand, this is the best available evidence, and a sham-controlled study with MVD would raise serious ethical concerns. Because of the inclusion of case reports and small case series, there is a possibility of publication bias, which may have resulted in an overestimation of the success rate that was found in this study. Unfortunately, the data were insufficient for performing formal statistical tests to assess publication bias.

Second, in this review, a global measurement of improvement was extracted from the included papers. This outcome is subjective (patient assessed) and it was not standardized in the vast majority of the included studies, as shown by our quality assessment. Unfortunately, we had to rely on these unstandardized self-assessed outcomes, and this is a significant limitation of the present study. Obviously, standardized outcome measurement such as validated tinnitus questionnaires should be used in future research, to gain better evidence of the true success rate. De Ridder et al. published an article describing the results of MVD for tinnitus using preoperative and postoperative questionnaires (e.g., visual analog scale and tinnitus questionnaire). ${ }^{12}$ However, these outcome measures could not be included in our analysis, because they could not be translated to global measurement of improvement, as used in all of the other studies.

Third, all included patients had an objectified NVC during surgery, and all patients underwent MVD of the cochleovestibular nerve. However, inclusion criteria for surgery varied considerably across the studies. Some patients were operated on based primarily on their disease history (e.g., intractable tinnitus/vertigo), with or without the suspicion of NVC on imaging or abnormal ABR, whereas others were operated on based on more specific diagnoses (e.g., typewriter tinnitus or disabling positional vertigo). Considering that asymptomatic NVCs are often reported, ${ }^{9}$ one should keep in mind that a proportion of patients might have undergone MVD for an incorrect indication, leading to a lower overall success rate.

Finally, an important limitation is that we were not able to collect IPD of the 2 largest studies describing MVD for patients with tinnitus $(\mathrm{n}=72)$ and MVD for patients with disabling vertigo $(n=163){ }^{33,34}$ Due to these missing data (ranging from $6 \%$ to $85 \%$ ), we were not able to perform a multivariate logistic regression analysis. Therefore, the conclusions from our IPD meta-analysis are based only on a univariate analysis and should be interpreted with caution. A larger sample size is needed to perform a multivariate logistic regression analysis and to gain more insight into the prognostic factors for successful surgery.

\section{Conclusions}

This systematic review and meta-analysis demonstrated 


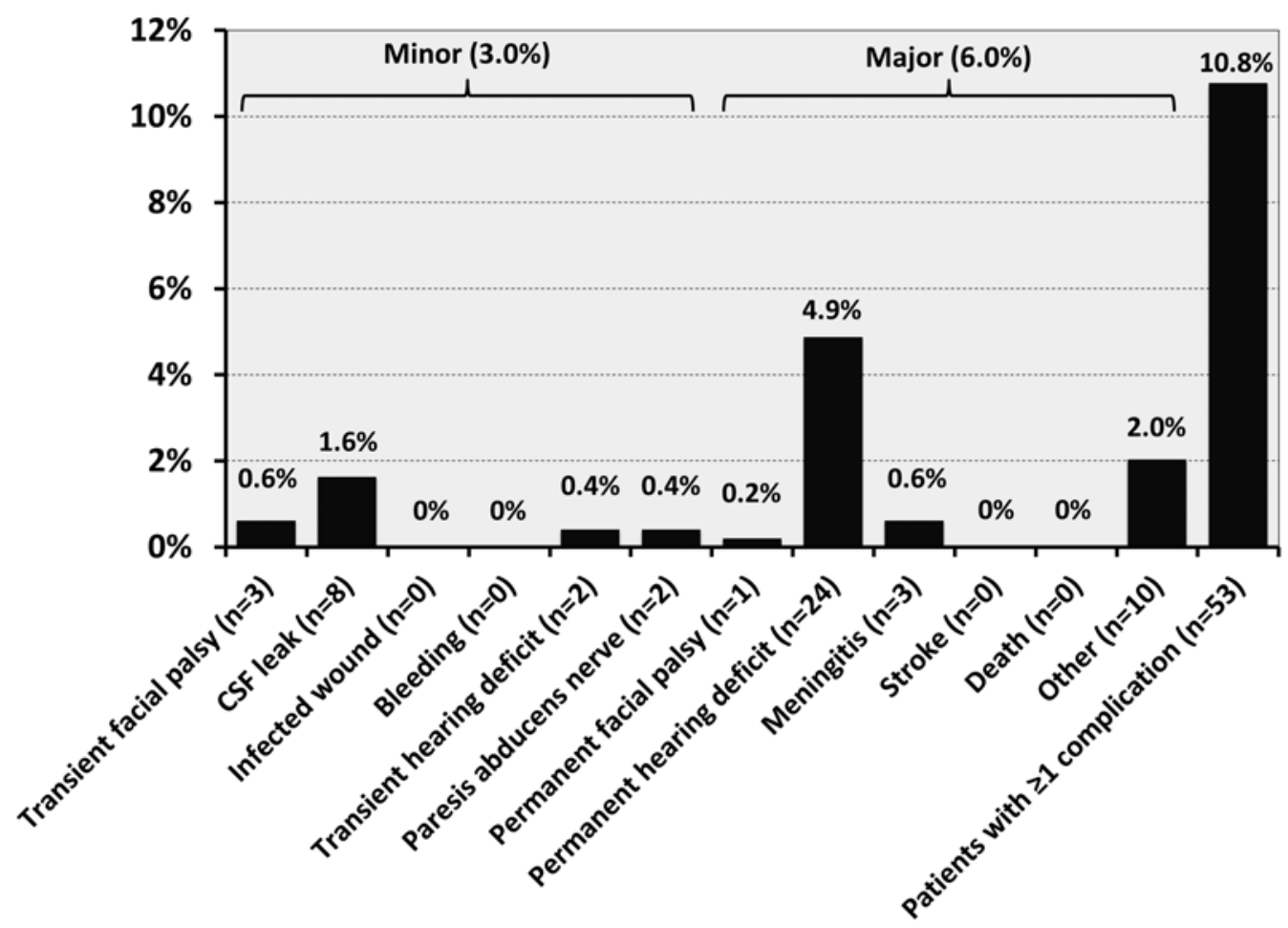

FIG. 4. Complication rates of MVD surgery of the cochleovestibular nerve. Only 22 of 35 articles (representing 492 patients) reported if there were complications and, if so, which ones. The "Other" category included epidural hematoma $(n=1)$, temporary vagal nerve paresis $(n=1)$, herpes zoster $(n=1)$, loss of vestibular function $(n=1)$, temporary vocal cord weakness $(n=1)$, temporary trochlear nerve paresis $(n=1)$, temporary bulbar paresis $(n=1)$, temporary swallowing problems $(n=1)$, transient cerebellar sign $(n=1)$, and cerebellar hematoma $(n=1)$.

a low success rate of MVD of the cochleovestibular nerve for treatment of tinnitus and vertigo. Also, a surgical complication rate of $11 \%$ was encountered. Therefore, this surgery cannot be considered a standard treatment method for either tinnitus or vertigo complaints. However, in patients

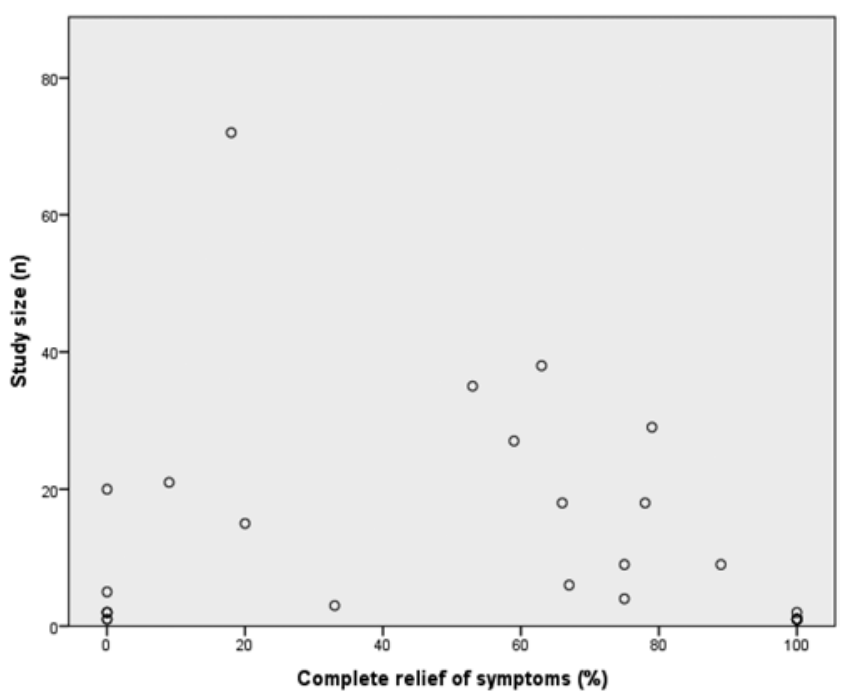

FIG. 5. Scatterplot of "sample size of study" versus "percentage of complete relief" to assess publication bias. with both tinnitus and vertigo, there was a substantially higher chance of treatment success. It is the combination of symptoms that suggests that an NVC is the underlying pathology and thus MVD might be appropriate. However, this systematic review was based on studies that had low levels of evidence; hence no definite recommendations can be made. Further validation is necessary to evaluate whether patients with combined symptoms are indeed better candidates for MVD.

\section{Acknowledgments}

We acknowledge S. van der Werf for helping us to compose our search strategy for MEDLINE and EMBASE. Moreover, we thank Y. A. Bayazit and T. Okamura, authors of included studies, for providing us their additional patient data.

\section{References}

1. Abo-Zaid G, Guo B, Deeks JJ, Debray TP, Steyerberg EW, Moons KG, et al: Individual participant data meta-analyses should not ignore clustering. J Clin Epidemiol 66:865-873, 873.e1-873.e4, 2013

2. Artz GJ, Hux FJ, Larouere MJ, Bojrab DI, Babu S, Pieper DR: Endoscopic vascular decompression. Otol Neurotol 29:995-1000, 2008

3. Bayazit YA, Catli T, Goksu N: Endoscopy assisted microvascular decompression for vascular conflict syndromes in 22 patients. Int Adv Otol 6:316-319, 2010 
4. Bejjani GK, Sekhar LN: Repositioning of the vertebral artery as treatment for neurovascular compression syndromes. Technical note. J Neurosurg 86:728-732, 1997

5. Borghei-Razavi H, Darvish O, Schick U: Disabling vertigo and tinnitus caused by intrameatal compression of the anterior inferior cerebellar artery on the vestibulocochlear nerve: a case report, surgical considerations, and review of the literature. J Neurol Surg Rep 75:e47-e51, 2014

6. Brackmann DE, Kesser BW, Day JD: Microvascular decompression of the vestibulocochlear nerve for disabling positional vertigo: the House Ear Clinic experience. Otol Neurotol 22:882-887, 2001

7. Brantberg K: Paroxysmal staccato tinnitus: a carbamazepine responsive hyperactivity dysfunction symptom of the eighth cranial nerve. J Neurol Neurosurg Psychiatry 81:451-455, 2010

8. Brookes GB: Vascular-decompression surgery for severe tinnitus. Am J Otol 17:569-576, 1996

9. Chadha NK, Weiner GM: Vascular loops causing otological symptoms: a systematic review and meta-analysis. Clin Otolaryngol 33:5-11, 2008

10. Dandy WE: Concerning the cause of trigeminal neuralgia. Am J Surg 24:447-455, 1934

11. De Ridder D, Heijneman K, Haarman B, van der Loo E: Tinnitus in vascular conflict of the eighth cranial nerve: a surgical pathophysiological approach to ABR changes. Prog Brain Res 166:401-411, 2007

12. De Ridder D, Vanneste S, Adriaenssens I, Lee AP, Plazier M, Menovsky T, et al: Microvascular decompression for tinnitus: significant improvement for tinnitus intensity without improvement for distress. A 4-year limit. Neurosurgery 66:656-660, 2010

13. Fries G, Perneczky A: Endoscope-assisted brain surgery: part 2-analysis of 380 procedures. Neurosurgery 42:226-232, 1998

14. Fuse T, Møller MB: Delayed and progressive hearing loss after microvascular decompression of cranial nerves. Ann Otol Rhinol Laryngol 105:158-161, 1996

15. Guevara N, Deveze A, Buza V, Laffont B, Magnan J: Microvascular decompression of cochlear nerve for tinnitus incapacity: pre-surgical data, surgical analyses and longterm follow-up of 15 patients. Eur Arch Otorhinolaryngol 265:397-401, 2008

16. Herzog JA, Bailey S, Meyer J: Vascular loops of the internal auditory canal: a diagnostic dilemma. Am J Otol 18:26-31, 1997

17. Higgins JPT, Green S (eds): Cochrane Handbook for Systematic Reviews of Interventions, Version 5.1.0. London: The Cochrane Collaboration, 2011 (http://handbook.cochrane.org) [Accessed September 29, 2016]

18. Isu T, Ito T, Murai H, Yamamoto K: Paroxysmal tinnitus and nystagmus accompanied by facial spasm. Surg Neurol 23:183-186, 1985

19. Jannetta PJ: Neurovascular compression in cranial nerve and systemic disease. Ann Surg 192:518-525, 1980

20. Jannetta PJ, Møller MB, Møller AR: Disabling positional vertigo. N Engl J Med 310:1700-1705, 1984

21. Kandan SR, Khan S, Jeyaretna DS, Lhatoo S, Patel NK, Coakham HB: Neuralgia of the glossopharyngeal and vagal nerves: long-term outcome following surgical treatment and literature review. Br J Neurosurg 24:441-446, 2010

22. Ko Y, Park CW: Microvascular decompression for tinnitus. Stereotact Funct Neurosurg 68:266-269, 1997

23. Kudo T, Ito K: Microvascular decompression of the eighth cranial nerve for disabling tinnitus without vertigo: a case report. Neurosurgery 14:338-340, 1984

24. Landis JR, Koch GG: The measurement of observer agreement for categorical data. Biometrics 33:159-174, 1977

25. Leclercq TA, Hill CL, Grisoli F: Retromastoid microsurgical approach to vascular compression of the eighth cranial nerve. Laryngoscope 90:1011-1017, 1980

26. Levine RA: Typewriter tinnitus: a carbamazepine-responsive syndrome related to auditory nerve vascular compression. ORL J Otorhinolaryngol Relat Spec 68:43-47, 2006

27. Mathiesen T, Brantberg K: Microvascular decompression for typewriter tinnitus-case report. Acta Neurochir (Wien) 157:333-336, 2015

28. McCabe BF, Gantz BJ: Vascular loop as a cause of incapacitating dizziness. Am J Otol 10:117-120, 1989

29. Meaney JF, Miles JB, Mackenzie IJ: Imaging of neurovascular compression in tinnitus. Lancet 344:200-201, 1994

30. Meyerhoff WL, Mickey BE: Vascular decompression of the cochlear nerve in tinnitus sufferers. Laryngoscope 98:602604, 1988

31. Miller LE, Miller VM: Safety and effectiveness of microvascular decompression for treatment of hemifacial spasm: a systematic review. Br J Neurosurg 26:438-444, 2012

32. Møller MB, Møller AR: Vascular compression syndrome of the eighth nerve. Clinical correlations and surgical findings. Neurol Clin 8:421-439, 1990

33. Møller MB, Møller AR, Jannetta PJ, Jho HD: Vascular decompression surgery for severe tinnitus: selection criteria and results. Laryngoscope 103:421-427, 1993

34. Møller MB, Møller AR, Jannetta PJ, Jho HD, Sekhar LN: Microvascular decompression of the eighth nerve in patients with disabling positional vertigo: selection criteria and operative results in 207 patients. Acta Neurochir (Wien) 125:7582,1993

35. Ohashi N, Yasumura S, Nakagawa H, Mizukoshi K, Kuze S: Vascular cross-compression of the VIIth and VIIIth cranial nerves. J Laryngol Otol 106:436-439, 1992

36. Okamura T, Kurokawa Y, Ikeda N, Abiko S, Ideguchi M, Watanabe K, et al: Microvascular decompression for cochlear symptoms. J Neurosurg 93:421-426, 2000

37. Pirayesh Islamian A, Lütjens G, Krauss JK: Microvascular decompression of the eighth cranial nerve for unilateral pulsatile tinnitus. Clin Neurol Neurosurg 117:102-106, 2014

38. Roland PS, Fell W, Meyerhoff W: Surgical decompression of the eighth nerve for tinnitus. Int Tinnitus J 1:139-146, 1995

39. Ryu H, Yamamoto S, Sugiyama K, Nishizawa S, Nozue M: Neurovascular compression syndrome of the eighth cranial nerve. Can the site of compression explain the symptoms? Acta Neurochir (Wien) 141:495-501, 1999

40. Sakaki T, Morimoto T, Miyamoto S, Kyoi K, Utsumi S, Hyo Y: Microsurgical treatment of patients with vestibular and cochlear symptoms. Surg Neurol 27:141-146, 1987

41. Schwaber MK, Hall JW: Cochleovestibular nerve compression syndrome. I. Clinical features and audiovestibular findings. Laryngoscope 102:1020-1029, 1992

42. Stewart LA, Clarke M, Rovers M, Riley RD, Simmonds M, Stewart G, et al: Preferred reporting items for systematic review and meta-analyses of individual participant data: The PRISMA-IPD statement. JAMA 313:1657-1665, 2015

43. Strupp M, von Stuckrad-Barre S, Brandt T, Tonn JC: Teaching neuroimages: Compression of the eighth cranial nerve causes vestibular paroxysmia. Neurology 80:e77, 2013

44. Tanrikulu L, Scholz T, Nikoubashman O, Wiesmann M, Clusmann H: Preoperative MRI in neurovascular compression syndromes and its role for microsurgical considerations. Clin Neurol Neurosurg 129:17-20, 2015

45. Vasama JP, Moller MB, Moller AR: Microvascular decompression of the cochlear nerve in patients with severe tinnitus. Preoperative findings and operative outcome in 22 patients. Neurol Res 20:242-248, 1998

46. Wuertenberger CJ, Rosahl SK: Vertigo and tinnitus caused by vascular compression of the vestibulocochlear nerve, not intracanalicular vestibular schwannoma: review and case presentation. Skull Base 19:417-424, 2009 
47. Yap L, Pothula VB, Lesser T: Microvascular decompression of cochleovestibular nerve. Eur Arch Otorhinolaryngol 265:861-869, 2008

48. Zakrzewska JM, Linskey ME: Trigeminal neuralgia. BMJ Clin Evid 2014:2014, 2014

49. Zhang L, Yu Y, Yuan Y, Xu J, Xu X, Zhang J: Microvascular decompression of cochleovestibular nerve in patients with tinnitus and vertigo. Neurol India 60:495-497, 2012

\section{Disclosures}

The authors report no conflict of interest concerning the materials or methods used in this study or the findings specified in this paper.

\section{Author Contributions}

Conception and design: van den Berge, Free. Acquisition of data: van den Berge, Posthumus. Analysis and interpretation of data: van den Berge, JMC van Dijk, Smidt, P van Dijk, Free. Drafting the article: van den Berge. Critically revising the article: JMC van Dijk, Smidt, P van Dijk. Reviewed submitted version of manuscript: van den Berge, JMC van Dijk, Smidt, P van Dijk, Free. Approved the final version of the manuscript on behalf of all authors: van den Berge. Statistical analysis: van den Berge, Smidt.

\section{Supplemental Information}

Online-Only Content

Supplemental material is available with the online version of the article.

Appendix. https://thejns.org/doi/suppl/10.3171/2016.8. JNS16992.

\section{Correspondence}

Minke J. C. van den Berge, Universitair Medisch Centrum Groningen, Afdeling KNO-heelkunde, BB20, Hanzeplein 1, 9700 RB

Groningen, The Netherlands. email: m.j.c.van.den.berge@umcg.nl. 